\title{
BMJ Open Effects of dry cupping on pain, function and quality of life in women with knee osteoarthritis: a protocol for a sham- controlled randomised trial
}

\author{
Nayara Silva Pontes (D , ,' Germanna Medeiros Barbosa (D) , \\ Hugo Jário Almeida Silva (D) , 'Rodrigo Scattone Silva (D) ,' \\ Clécio Gabriel Souza (1) ,' Caio Alano de Almeida Lins (1) ,' \\ Marcelo Cardoso de Souza (1) ${ }^{1}$
}

To cite: Pontes NS, Barbosa GM, Almeida Silva HJ, et al. Effects of dry cupping on pain, function and quality of life in women with knee osteoarthritis: a protocol for a sham-controlled randomised trial. BMJ Open 2020;10:e039857. doi:10.1136/ bmjopen-2020-039857

- Prepublication history for this paper is available online. To view these files, please visit the journal online (http://dx.doi. org/10.1136/bmjopen-2020039857).

Received 28 April 2020 Revised 19 November 2020 Accepted 27 November 2020

D) Check for updates

(c) Author(s) (or their employer(s)) 2020. Re-use permitted under CC BY-NC. No commercial re-use. See rights and permissions. Published by BMJ.

${ }^{1}$ Postgraduate Program in Rehabilitation Sciences, Faculty of Health Sciences of Trairi,

Federal University of Rio Grande do Norte (UFRN/FACISA), Santa Cruz, Brazil

${ }^{2}$ Faculty of Health Sciences of Trairi, Federal University of Rio Grande do Norte (UFRN/FACISA), Santa Cruz, Brazil

Correspondence to Professor Marcelo Cardoso de Souza;

marcellogv@hotmail.com

\section{ABSTRACT}

Introduction Knee osteoarthritis (KOA) is the most common cause of pain and disability worldwide. Dry cupping has been used as non-pharmacological approach to control pain and improve physical function. However, there is a lack of high-quality scientific evidence regarding its effects on this condition. This protocol describes a sham-controlled, randomised and simple blind study that aims to evaluate the effect of dry cupping on pain, function and quality of life in women with KOA.

Methods and analysis Sixty-two women diagnosed with KOA, based on American College of Rheumatology clinical criteria, and aged from 50 to 75 years, will be randomly distributed into two groups (31 per group): real and sham dry cupping. Both applications will occur with acrylic cups around the knee. The intervention will last 15 min, two times a week over six consecutive weeks, for a total of 12 sessions. Both groups will be assessed at four different times: before the intervention (T0), after 3 weeks intervention (T3), at the end of the protocol (T6) and 4 weeks after the interventions (follow-up: T10). The primary outcome will be pain intensity (Numerical Pain Rating Scale), and secondary outcomes will be knee-related health status (Western Ontario and McMaster Universities Osteoarthritis Index), functional capacity (8-step stair climb test, 40-metre fast-paced walk test and 30-second chair stand test), quality of life (Short-Form 36) and global perceived effect.

Ethics and dissemination This protocol was approved by the UFRN/FACISA Ethics Committee (number 3.737.688). The study results will be disseminated to the participants and submitted to a peer-reviewed journal and scientific meetings.

Trial registration number NCT04331158.

\section{BACKGROUND}

Knee osteoarthritis (KOA) is the most common chronic musculoskeletal condition, and a major cause of pain and functional disability worldwide. ${ }^{12}$ Given its progressive nature, it is estimated that there has been a $104.9 \%$ increase in disability-adjusted life
Strengths and limitations of this study

- There is no standardised protocol in the literature for the use of dry cupping in individuals with knee osteoarthritis (KOA)

- This is the first study to use a sham dry cupping group in individuals with $\mathrm{KOA}$, with concealed allocation and blinding of the outcome assessor and biostatistician.

- This study will demonstrate the effect of the shortterm and medium-term application (1-month followup after interventions) of dry cupping in individuals with KOA.

- The therapist who will deliver the dry cupping or sham interventions will not be blinded.

- Participants will not present with radiographically confirmed KOA. They will, however, meet the American College of Rheumatology clinical criteria and have a minimum level of pain in order to ensure the possibility for improvement with the interventions.

years over a period of 26 years. ${ }^{3}$ Although KOA affects both sexes, women over the age of 50 years exhibit a higher prevalence $(44 \%-70 \%) .{ }^{4}$ The high chronicity, disability and prevalence associated with the disease results in a considerable economic load for the patient and significant pressure on healthcare systems worldwide..$^{5-7}$

According to the guidelines and recommendations of important societies such as the Osteoarthritis Research Society International (OARSI) ${ }^{8}$ European League Against Rheumatism, ${ }^{9}$ American College of Rheumatology $(\mathrm{ACR})^{10}$ and American Academy of Orthopedic Surgeons, ${ }^{11}$ the treatment of KOA involves pharmacological, nonpharmacological and as a last resort, surgical interventions. As part of non-pharmacological treatment complementary to KOA, cupping 
therapy is currently used to control the symptoms of this population. ${ }^{12}$

Cupping is a long-used therapy applied worldwide to treat chronic pain. ${ }^{12}{ }^{13}$ Among the different application methods, dry cupping is the most commonly used in clinical practice, primarily because no scarification is involved. ${ }^{12-14}$ The technique consists of placing a plastic, glass or silicone cup on the skin, normally in painful areas or at acupuncture points, using a suction pump to generate negative pressure in the region, creating a vacuum in subcutaneous tissues. ${ }^{12}$ Cupping application time in KOA varies between 5 and $10 \mathrm{~min}$, and may extend up to $20 \mathrm{~min}$, associated with the force of two suctions. ${ }^{15} 16$ Bruising may appear after applications, but usually disappears in a few days, requiring a time period between sessions for tissue recovery. ${ }^{14}$ Despite being a complementary resource in the treatment of individuals with KOA, the mechanisms of action and clinical effects of cupping therapy are poorly understood. ${ }^{17}$ In addition, there are no literature protocols of well-designed standardised interventions in this population. ${ }^{18}$

There are currently two systematic reviews on cupping therapy in KOA that reported significant heterogeneity between studies, with a high risk of bias. ${ }^{15} 16$ In the vast majority, the assessor and participants were not blinded, exhibited inadequate randomisation, did not involve a follow-up, limited interventions to 1 month and did not use a sham dry cupping group for comparison purposes. ${ }^{1516}$ Thus, well-designed trials are needed before any conclusions can be drawn about the efficacy of cupping therapy in KOA.

As such, the aim of this study will be to assess the effects of dry cupping on pain, function and quality of life in individuals with KOA. Based on unpublished data from our recent protocol ${ }^{19}$ performed in people with chronic pain, the scientific hypothesis of this study will be that both the dry and sham cupping interventions will improve pain, function and quality of life in this population.

\section{METHOD}

\section{Design}

The study offers a protocol for a simple blind placebocontrolled randomised clinical trial, registered on the clinical trial platform and approved by the research ethics committee (protocol number: 3.737.688) of the Trairi School of Health Sciences-Federal University of Rio Grande do Norte (FACISA/UFRN). Participants will be randomised into two groups, each containing 31 individuals: one group will receive classic dry cupping and the other sham dry cupping (figure 1).

The study will follow Consolidated Standards of Reporting Trials guidelines, ${ }^{20}$ and will be based on the OARSI consensus for recommended clinical trials in $\mathrm{KOA}^{21}$ and the Standards for Reporting Interventions in Clinical Trials of Cupping. ${ }^{22}$ Moreover, the protocol follows Standard Protocol Items : Recommendations for
Interventional Trials ${ }^{23}$ and the Template for Intervention Description and Replication. ${ }^{24}$

\section{Participants}

Sixty-two women with diagnosis of KOA will be recruited based on the ACR clinical criteria ${ }^{25}$ : (1) crepitus (present), morning stiffness $>30$ min and bony enlargement of the knee (present) or (2) crepitus (present) and morning stiffness $\leq 30 \mathrm{~min}$ or (3) crepitus (absent) and bony tenderness (present).

The volunteers will be recruited by advertising the research project on a local radio station and from the patient waiting list of the FACISA/UFRN Physiotherapy School Clinic. They will be contacted by telephone to clarify any doubts and conduct the first screening for inclusion. After being informed of the study objectives and procedures, eligible individuals provided written informed consent.

Personal information (name, address, telephone number, profession, schooling level and income), anthropometric data (age, height, weight and body mass index $[\mathrm{BMI}])$ and clinical history of the disease will be collected. Participants' personal data will be numerically coded in a database, to which only the researcher in charge of randomisation and blinding will have access.

\section{Inclusion criteria}

- Women aged between 50 and 75 years. ${ }^{26}$

- Diagnosed with KOA based on ACR clinical criteria. ${ }^{25}$

- Pain intensity in the knee between 3 and 8, according to the Numerical Pain Rating Scale (NPRS). ${ }^{27}$

- BMI less than $35 \mathrm{~kg} / \mathrm{m}^{2}{ }^{28}$

\section{Exclusion criteria}

- Having undergone physiotherapy treatment for the knee in the 3 months before the trial. ${ }^{28}$

- Being engaged in $>45 \mathrm{~min} /$ week of moderate to vigorous physical activity. ${ }^{28}$

- Having been submitted to the cupping application and/or intervention. ${ }^{19}$

- Having used corticosteroid knee injection in the previous 6 months. ${ }^{28}$

- Exhibiting any medical restriction that hindered their participation in the assessments and interventions proposed (cardiorespiratory, neurological and/or musculoskeletal changes). ${ }^{28}$

- Diagnosed with fibromyalgia, rheumatoid arthritis or any systemic inflammatory arthritis. ${ }^{29}$

- Exhibiting ligament or clinically diagnosed meniscal injury or with positive results in the Apley or anterior/ posterior drawer tests. ${ }^{30}$

- Having undergone previous ankle, knee or hip surgery. ${ }^{31} 32$

- Clinical diagnosis of dementia or inability to complete the study questionnaires. ${ }^{32}$

- Any contraindication to the use of cupping therapy (cutaneous injury region where it will be applied, 
cancer, kidney, liver or heart failure, pacemaker or pregnancy). ${ }^{18}$

- Uncontrolled diabetes or hypertension. ${ }^{30}$

- Having a trip scheduled in the 2 months following the start of the study. ${ }^{19}$

\section{Research team}

This study will include five researchers, one in charge of randomising the groups, one to conduct all the assessments, two for the interventions and one for statistical analyses.

\section{Randomisation and blinding}

The participants selected at screening who provide written informed consent will be randomly allocated to two groups: one will be submitted to dry cuppingapplying acrylic cups around the ankle, and the other to sham dry cupping-with acrylic cups containing small holes to release suction after a few seconds. ${ }^{33}$
In order to avoid selection bias, the concealed allocation method will use consecutively numbered opaque sealed envelopes. The randomisation process will be conducted by an independent researcher who will not be involved in other study procedures, using the www.randomization. com website. Each participant's random allocation will be revealed just before the intervention.

All the participants will be informed of the study aim to test two different techniques: classic dry cupping versus sham dry cupping. A number of measures will be adopted to ensure blinding is not compromised. Participants will be instructed not to reveal which intervention they received. To that end, different intervention times will be assigned to the groups, thereby reducing contact between participants. They will also be asked to use comfortable pants during reassessments to avoid exposing the knee to bruising after dry cupping application. All the assessments will be conducted by a researcher who will not be involved

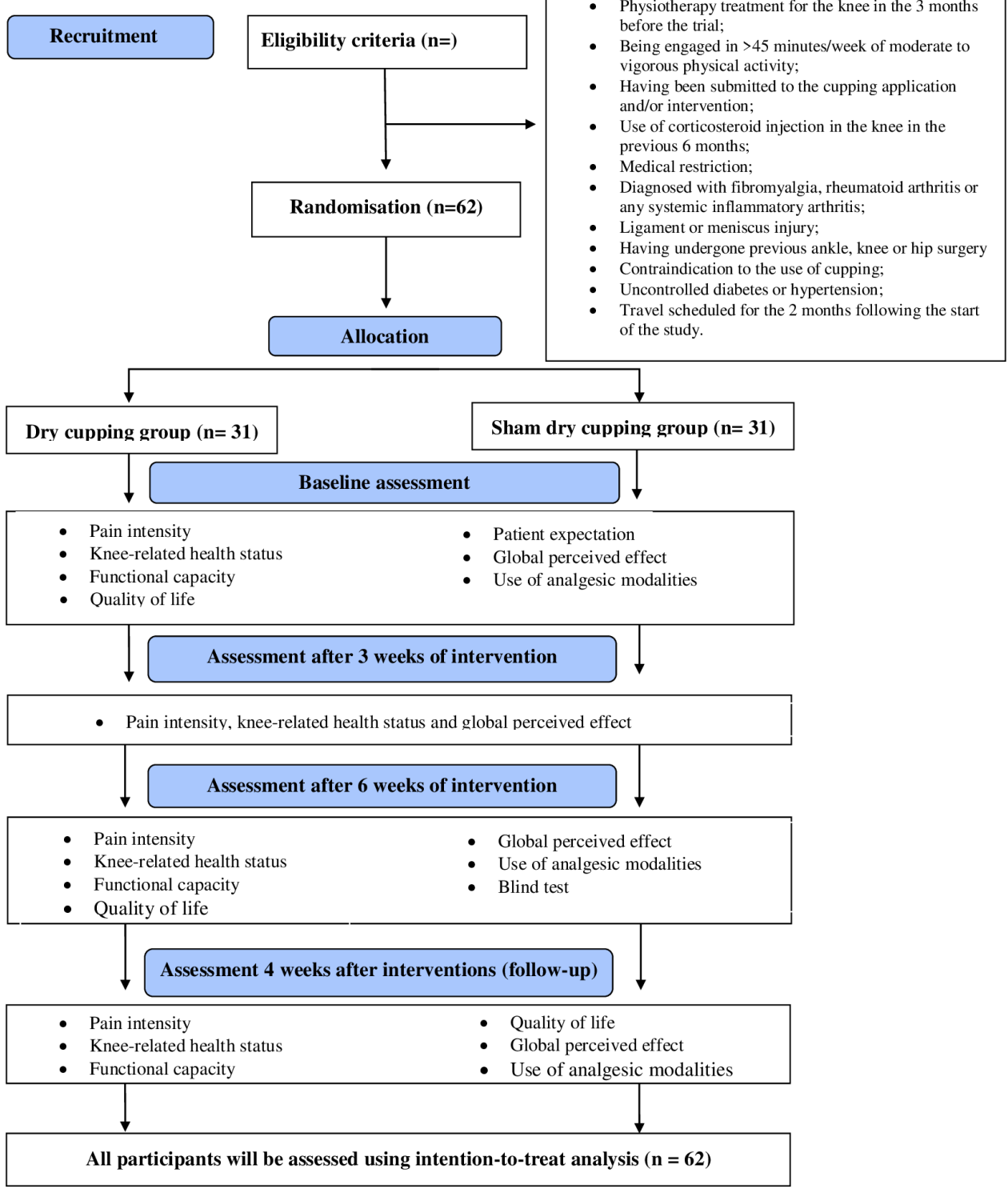

Figure 1 Design and flow of participants through the trial. 
in the interventions and will remain blind to the treatment groups. The results of assessments and functional tests will not be divulged to the researchers in charge of interventions. At the end of the protocol, each participant will be asked which intervention they believe they received, in order to determine the efficacy of participant blinding. However, the identity of the group will only be revealed to the participant after the last follow-up assessment. Finally, the researcher in charge of statistical analysis will not take part in the assessments or interventions and will receive the data without the subjects or groups being identified.

\section{Procedures}

Participants will be submitted to initial assessment (T0), which will consist of clinical and functional aspects (pain intensity, knee-related health status, functional capacity, quality of life, treatment expectation and overall patient status) related to KOA. If both knees exhibit KOA, the limb with the highest score on the NPRS will be submitted to intervention.

On the same day, participants will receive their respective interventions after being allocated to the dry or sham cupping group. A second assessment (T3) will occur after 3 weeks of interventions to analyse pain, knee-related health status and overall patient status. After 6 weeks, at the end of the proposed treatment, participants will undergo a third assessment (T6), at which time the same variables evaluated at baseline will be collected, except treatment expectation, and the efficacy of participant blinding will be assessed. ${ }^{34}$ The fourth assessment (T10) will be carried out 4 weeks after the interventions (follow-up), where all the variables evaluated in $\mathrm{T} 6$ will be collected.

Before the start of each intervention session, participants will be asked whether they received any therapy to control knee pain or in other parts of the body, including analgesic or anti-inflammatory medication, cryotherapy, heat or any other non-pharmacological or analgesic modality.

The flowchart of the study is shown in figure 1.

\section{Interventions}

Interventions will be carried out at a physiotherapy School Clinic of the University, in an air-conditioned room with a pleasant ambient temperature. The patients will be attended individually by two experienced physiotherapists qualified to apply dry cupping. Before application, all the participants will be informed about the suction sensation, the likely bruising that will occur and the possible adverse effects described in the literature. ${ }^{35}$ In the event of any severe adverse effect, the participant will be referred to a doctor. A manual suction pump and five acrylic cups will be used for the applications (Dong Yang).

In both interventions, the subjects will remain in dorsal decubitus on a gurney, with a wedge maintaining the torso reclined for better comfort. A roll will be placed on the posterior region of the knee to maintain it at a $15^{\circ}$ of flexion $\left(0^{\circ}=\right.$ total knee extension $)$. Finally, an opaque structure will be placed transversally on the subject's thighs, such that they will not be able to see the technique being applied (figure 2).

\section{Dry cupping group}

The recommendations described in previous studies will be used to apply dry cupping. ${ }^{33}{ }^{35-41}$ The protocol will consist of two weekly interventions, over six consecutive weeks. Dry cupping will be applied with the force of two suctions, generating negative pressure mild (between 100 and less than 300 millibars) on the skin for $15 \mathrm{~min}$. If any participant still reports discomfort when applying small suction cups with (2.3) internal diameter, we will adjust to a comfortable pressure by applying only a suction (figure 3). ${ }^{33} 3538$ The dry cupping application sites will be the same as their acupuncture counterparts, in line with earlier studies. ${ }^{3639-41}$ The intervention protocol is described in box 1 .

\section{Sham dry cupping group}

Sham dry cupping group subjects received the same procedures as the dry cupping group, the difference being that the negative pressure imposed after application will be released in a few seconds ( $\sim 3 \mathrm{~s})$ (figure 4$)$. To that end, the cups will be prepared with small holes $(<2$ $\mathrm{mm}$ in diameter). ${ }^{19} 33$ A double-sided transparent adhesive tape will secure the acrylic cups to the patient's skin. That way the suction cups will be attached to the skin without any negative pressure and without any effect. Dry cupping sham will also consist of two weekly interventions

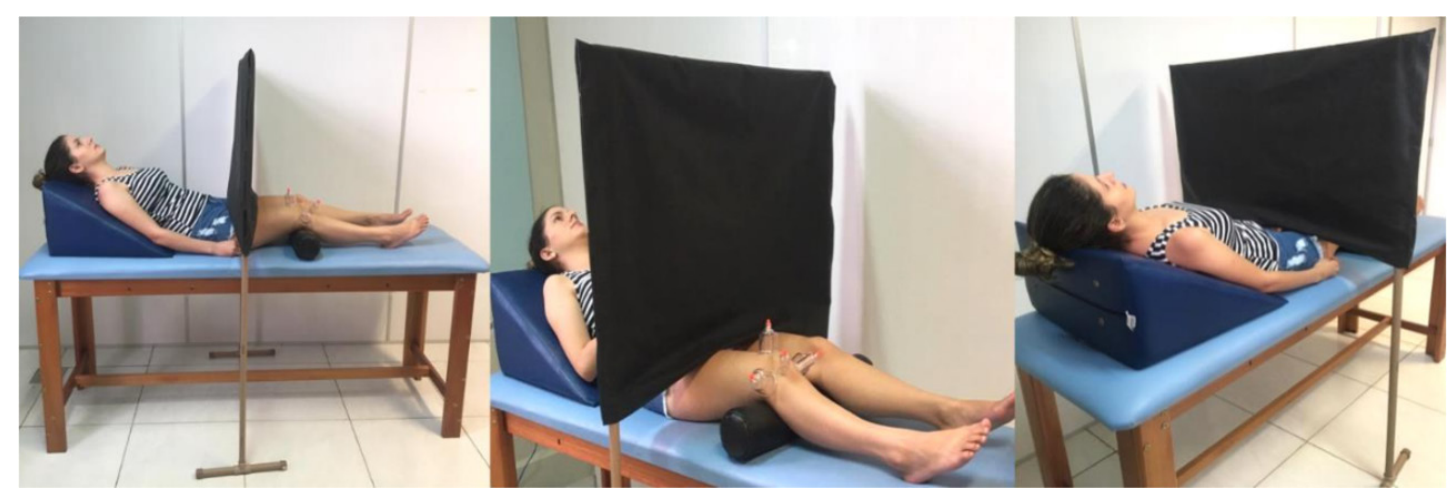

Figure 2 Participant position during interventions. 


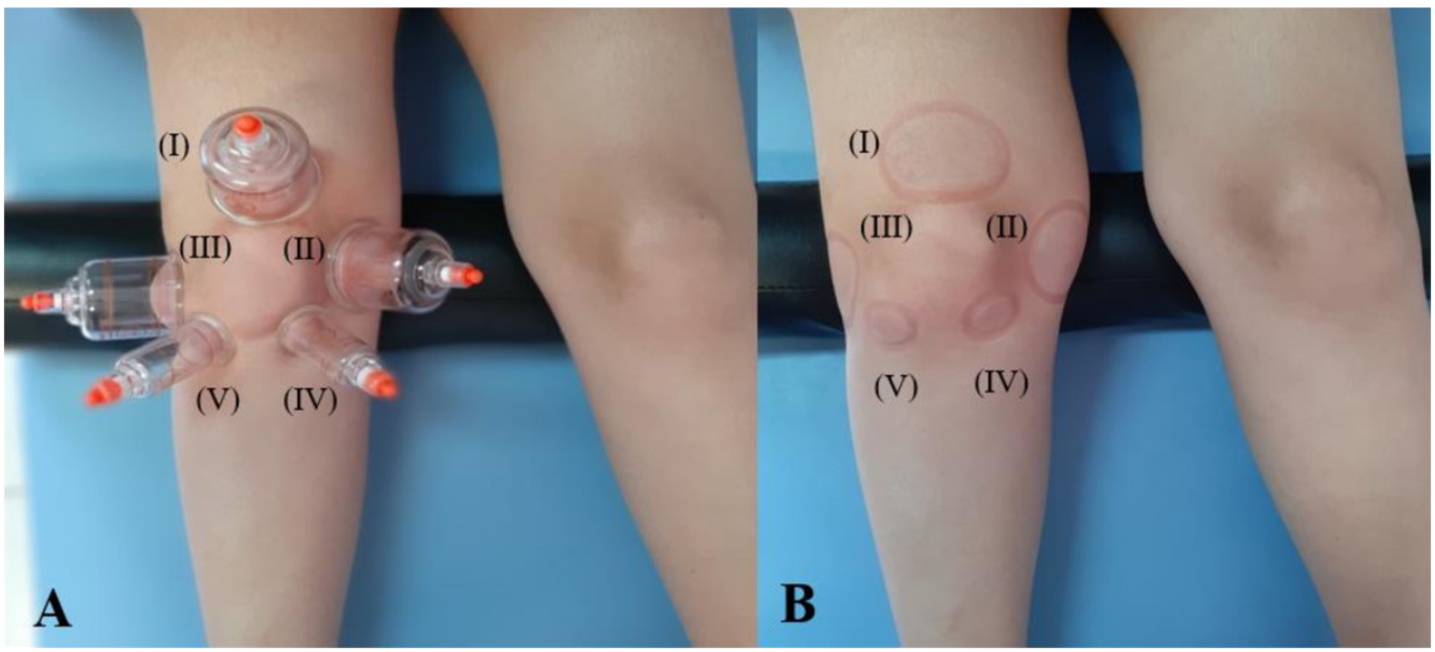

Figure 3 Dry cupping sites during $(A)$ and after $(B)$ intervention. Application in the suprapatellar region, Heding acupuncture site (I); in the medial region, Liver 8 acupuncture site (II); in the lateral region, GB 33 acupuncture point (III); in the inferior-medial region, medial Xivan (MN-LE-16) acupuncture point (IV); in the inferior-lateral region, lateral Xiyan (MN-LE-16) acupuncture point (V).

over six consecutive weeks, and will be applied with the force of two suctions, generating negative pressure on the skin for $15 \mathrm{~min}$. The treatment protocol is described in box 1 .

\section{Assessments}

Figure 5 lists the primary and secondary outcomes, as well as assessment moments during the study.

\section{Primary outcome}

Pain intensity

Pain intensity will be measured by the NPRS, in which individuals classify their pain on an 11-point scale. This scale will be placed in front of the participant and will vary from 0 to 10 points, 0 representing the complete absence of pain and 10 the worst pain imaginable. ${ }^{27}$ Pain intensity will be assessed both at rest and after each functional test (30-second chair stand test [30 s-CST], 8-step stair climb test [8-step SCT] and 40-metre fast-paced walk test [40 m FPWT]).

\section{Secondary outcomes}

Knee-related health status

The Western Ontario and McMaster Universities Osteoarthritis Index (WOMAC) will be used to assess the kneerelated health status. The questionnaire, translated and validated for Portuguese and specific for assessing patients with KOA, contains 24 questions divided into three categories that assess pain, stiffness and self-reported physical function. ${ }^{42}$ Items are assessed by a Likert scale and each question is attributed a score between 0 and 96 points, according to the patient's answer, as follows: none $=0$; low $=1$; moderate $=2$; severe $=3$; very severe $=4$. The higher the score obtained, the more severe the pain, stiffness or physical function. ${ }^{43}$

\section{Functional capacity}

- 30 s-CST: this test will be conducted using an armless chair, with seat height of $43 \mathrm{~cm}$ and non-slip rubber on the four legs. The back of the chair will against a wall to avoid oscillations during the rising and sitting movements. The participant will sit in the middle of the chair, back straight, feet aligned with the shoulders and resting on a stable flat floor. The test will consist of rising from the chair as many times as possible for $30 \mathrm{~s}$. It will therefore be possible to assess different skill levels, with scores ranging from 0 , for those that could not complete a single repetition, to maximum values of 20 repetitions, for individuals with good physical conditioning. ${ }^{44}$ A 2 min rest period will be allowed between functional tests.

- 8-step SCT: in the stair climb test, participants will be placed in front of the stairway and at the researcher's signal, will climb eight steps and then descend, in the shortest time possible. The test will be conducted in a well-lit area free of traffic and external distractions, and each step will be $20 \mathrm{~cm}$ high. A pretest will be conducted to identify the need for safety measures. The final score will be calculated based on the length of time the subject requires to perform the procedure and compared with the normal test values. ${ }^{44}$

- 40 m FPWT: the $40 \mathrm{~m}$ FPWT will be performed over four $10 \mathrm{~m}$ laps (delimited by tape and cones), in which the participant will be instructed to cover 10 $\mathrm{m}$, go around a cone and repeat the $10 \mathrm{~m}$ course until walking $40 \mathrm{~m}$. The time will be recorded only between the initial and final markers. The final score will be based on the time the participant takes to complete the procedure and compared with normal values for healthy adults. ${ }^{44}$ 
Box 1 Treatment protocol applied to the two groups

\section{Dry cupping group-dry cupping application}

The cupping group participants will receive dry cupping therapy with five acrylic cups (three size 1 and two size 5 suction cups). Two different sizes will be used to cover a larger part of the area and ensure better fixation of the suction cups according to the knee anatomy (figure 3 ).

- One size 1 suction cup (internal diameter of $4.5 \mathrm{~cm}$ ), located just above the patella, at the Heding acupuncture point (l). ${ }^{40}$

- One size 1 suction cup (internal diameter of $4.5 \mathrm{~cm}$ ), located $5 \mathrm{~cm}$ from the medial patellar edge, at acupuncture point Liver 8 (II). ${ }^{37} 4041$

- One size 1 suction cup (internal diameter of $4.5 \mathrm{~cm}$ ), located $3 \mathrm{~cm}$ lateral to the patella, at acupuncture point GB 33 (III). ${ }^{374}$

- One size 5 suction cup (internal diameter of $2.3 \mathrm{~cm}$ ), located medially to the patellar tendon, below the patella, at the medial Xiyan (MN-LE-16)-inferior-medial acupuncture point (IV). ${ }^{36} 3739$

- One size 5 suction cup 5 (internal diameter of $2.3 \mathrm{~cm}$ ), located laterally to the tendon below the patella, at the lateral Xiyan (MNLE-16) - inferior-lateral acupuncture point (V). ${ }^{36} 3739$

\section{Sham dry cupping group-sham dry cupping application}

The sham dry cupping group will receive dry cupping application with five acrylic cups (three size 1 and two size 5 suction cups). Two different sizes will be used to cover a larger part of the area and ensure better fixation of the suction cups according to the knee anatomy. However, the cups will be prepared with small holes $(<2 \mathrm{~mm}$ in diameter) to release negative pressure in a few seconds and a double-sided transparent adhesive tape will be used to secure the acrylic cups on the patient's skin. That way the suction cups will be attached to the skin without any negative pressure and without any effect (figure 4).

- One size 1 suction cup (internal diameter of $4.5 \mathrm{~cm}$ ), located just above the patella, at the Heding acupuncture point (I). ${ }^{40}$

- One size 1 suction cup (internal diameter of $4.5 \mathrm{~cm}$ ), located $5 \mathrm{~cm}$ from the medial patellar edge, at acupuncture point Liver 8 (II). ${ }^{374041}$

- One size 1 suction cup (internal diameter of $4.5 \mathrm{~cm}$ ), located $3 \mathrm{~cm}$ lateral to the patella, at acupuncture point GB 33 (III). ${ }^{3741}$

- One size 5 suction cup (internal diameter of $2.3 \mathrm{~cm}$ ), located medially to the patellar tendon, below the patella, at the medial Xiyan (MN-LE-16) -inferior-medial acupuncture point (IV). ${ }^{36} 3739$

- One size 5 suction cup 5 (internal diameter of $2.3 \mathrm{~cm}$ ), located laterally to the tendon below the patella, at the lateral Xiyan (MNLE-16) - inferior-lateral acupuncture point (V). ${ }^{36} 3739$

\section{Quality of life}

The quality of life will be evaluated by the Portuguese version of the Short Form-36 (SF-36) questionnaire. The instrument consists of 36 questions and assesses eight different domains that influence quality of life, considering individuals' perception about aspects of their own health in recent weeks. Each item contains a group of answers on a graduated Likert scale, with the following dimensions: physical function, physical aspect, pain, overall health, vitality, social function, emotional aspect and mental health. Total score ranges from 0 to 100 , where the higher the value, the better the quality of life. ${ }^{45}$

\section{Patient expectation}

A 5-point Likert scale was applied to assess patient expectation in terms of the intervention. The scale will be placed in front of the participant, who will be asked the following question: "with dry cupping intervention do you think your situation will: (1) worsen considerably, (2) worsen a little, (3) neither improve nor worsen, (4) improve a little or (5) improve considerably. ${ }^{46}$

\section{Global perceived effect}

Global perceived effect (GPE) is a 11-point Likert scale (ranging from -5 to +5 ) that compares the patients' current health status with that at the onset of symptoms. Positive scores are attributed to individuals who improved when compared with baseline and negative scores to those who worsened. ${ }^{47} 48$

\section{Other therapeutic modalities}

Participants of both groups will be asked about whether they made use of any therapeutic modality for knee pain or pain in any other part of the body, including analgesic and/or anti-inflammatory drugs, ice, heat or other nonpharmacological modalities for pain relief during the study period.

\section{Blinding test}

Blinding will be assessed by asking patients which treatment they believe they underwent, that is, whether they feel they were allocated to real or sham cupping intervention. ${ }^{33}$

\section{Patient and public involvement}

Patients were not involved in the study design, the research question or recruiting procedures. At the end of the study, the results will be reported to the participants in the form of lectures, showing the effects found in the variables studied. If one technique is found to be superior, the most effective intervention will be guaranteed to the subjects.

\section{Researcher training}

A series of training stages will be implemented before the study to conduct assessments and interventions, in order to standardise the procedures and reach a consensus among the researchers involved.

\section{Sample size calculation}

Sample size was calculated based on the NPRS of a previous study. ${ }^{49} \mathrm{~A}$ statistical power of $80 \%$ was used to detect a minimum difference between the means of 1.7 points, with an estimated SD of 2.25 points and $5 \%$ significance level. To that end, 28 individuals per group will be needed. A $10 \%$ sample loss was added to this total, thereby requiring 31 individuals in both groups.

\section{Statistical analysis}

The statistical analyses will be performed by a blinded biostatistician using a commercial software. For statistical analysis, the independent variables of group (dry cupping and sham dry cupping) and time (T0, T3, T6 and T10) will be considered for each dependent variable: pain intensity, total WOMAC index, 30 s-CST, 8-step SCT, $40 \mathrm{~m}$ FPWT, SF-36 questionnaire and GPE. The ShapiroWilk test will be applied to verify data distribution and the 


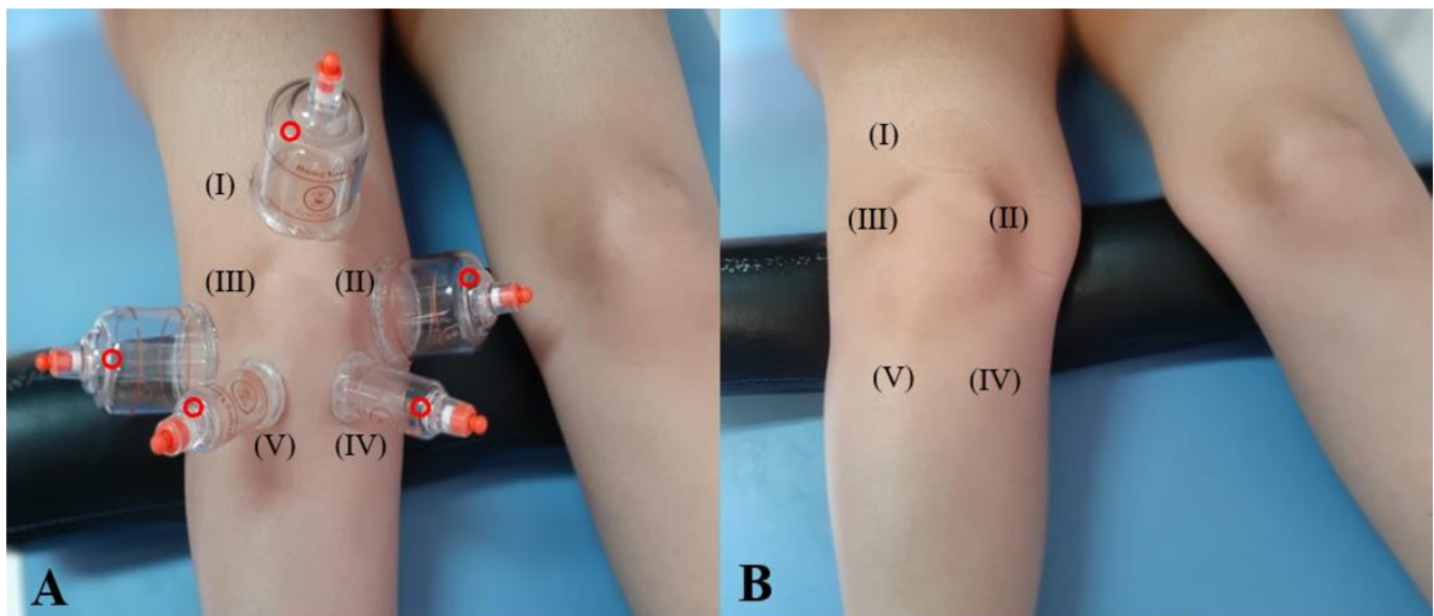

Figure 4 Sham dry cuppingsites during (A) and after (B) intervention. Application in the suprapatellar region, Heding acupuncture site (I); in the medial region, Liver 8 acupuncture site (II); in the lateral region, GB 33 acupuncture point (III); in the inferior-medial region, medial Xivan (MN-LE-16) acupuncture point (IV); in the inferior-lateral region, lateral Xiyan (MN-LE-16) acupuncture point $(\mathrm{V})$. The red circles demonstrate the sites with holes.

Levene test will be used to analyse homogeneity of variance. If the results are normally distributed, an analysis of variance with a mixed design will be conducted for the primary and secondary outcomes, with time as the withinsubject factor and group as the between subject factor. If the data are not normally distributed, Friedman test will be used. Sphericity will be tested using the Mauchly

\begin{tabular}{|c|c|c|c|c|c|}
\hline & \multicolumn{5}{|c|}{ STUDY PERIOD } \\
\hline & \multirow{2}{*}{$\begin{array}{c}\text { Screening } \\
\text { (-T1) }\end{array}$} & \multirow{2}{*}{$\begin{array}{c}\text { Baseline } \\
\text { (T0) }\end{array}$} & \multicolumn{3}{|c|}{ Post-allocation } \\
\hline TIMEPOINT & & & $\begin{array}{c}3 \text { weeks } \\
\text { (T3) }\end{array}$ & $\begin{array}{c}6 \text { weeks } \\
\text { (T6) }\end{array}$ & $\begin{array}{l}4 \text { weeks after } \\
\text { the end of the } \\
\text { interventions } \\
\text { (T10) }\end{array}$ \\
\hline \multicolumn{6}{|l|}{ ENROLMENT: } \\
\hline \multirow{2}{*}{$\begin{array}{l}\text { Eligibility screen } \\
\text { Informed consent }\end{array}$} & $x$ & & & & \\
\hline & $x$ & & & & \\
\hline Allocation & & $x$ & & & \\
\hline \multicolumn{6}{|l|}{ INTERVENTIONS: } \\
\hline \multicolumn{6}{|l|}{ Dry cupping ( $n=31$ ) } \\
\hline \multicolumn{6}{|l|}{$\begin{array}{l}\text { Sham Dry Cupping } \\
(n=31)\end{array}$} \\
\hline \multicolumn{6}{|l|}{ ASSESSMENTS: } \\
\hline \multirow{2}{*}{$\begin{array}{c}\text { Pain Intensity } \\
\text { Knee-related health } \\
\text { status }\end{array}$} & & $x$ & $x$ & $x$ & $x$ \\
\hline & & $x$ & $x$ & $x$ & $x$ \\
\hline Functional Capacity & & $x$ & & $x$ & $x$ \\
\hline \multirow{3}{*}{$\begin{array}{c}\text { Quality of Life } \\
\text { Patient Expectation } \\
\text { Global Perceived } \\
\text { Effect }\end{array}$} & & $x$ & & $x$ & $x$ \\
\hline & & $x$ & & & \\
\hline & & $x$ & $x$ & $x$ & $x$ \\
\hline $\begin{array}{l}\text { Register of usage of } \\
\text { other analgesic } \\
\text { modality }\end{array}$ & & $x$ & $x$ & $x$ & $x$ \\
\hline Blinding test & & & & $x$ & \\
\hline
\end{tabular}

Figure 5 Schedule of the study protocol according to the Standard Protocol Items: Recommendations for Interventional Trials checklist. test, and if the assumption of sphericity is violated, the Greenhouse-Geisser correction will be used. Time-bygroup interaction and intergroup and intragroup differences will be analysed for all the variables. Finally, the Bonferroni test will be used in post hoc analyses in order to determine whether differences exist between groups (dry cupping and sham dry cupping) in the different time points. An intention-to-treat analysis will be applied to ensure the effects of the randomisation, so that prognostic factors are evenly distributed in both groups. ${ }^{21} \mathrm{~A}$ $5 \%$ significance level and $95 \%$ CI will be adopted for all statistical analyses.

\section{DISCUSSION}

KOA is a public health problem and one of the major cause of pain and functional incapacity. ${ }^{3}$ The present study aims to assess the effects of dry cupping on pain, function and quality of life of individuals with KOA. The protocol specifies intervention in two groups, one with dry cupping and another with sham dry cupping, both applied two times a week for six consecutive weeks, totaling 12 treatment sessions, and a follow-up 4 weeks after the interventions.

There is no well-defined protocol in terms of the amount of suction, number of interventions in 1 week and dry cupping application time in individuals with KOA. ${ }^{1516}$ In the literature, one study suggests two suctions for musculoskeletal pain and a 15 min application time, ${ }^{12}$ while another study suggests an application time of $5 \mathrm{~min}$, $10 \mathrm{~min}$ or more to reduce pain. ${ }^{35} \mathrm{~A}$ number of complications may occur and are described in the latter study when dry cupping application lasts more than 20 min. ${ }^{13}$ With respect to application frequency, the study using pulsatile dry cupping in individuals with KOA suggests two times a week. ${ }^{38}$

In the literature, the use of dry cupping therapy to treat individuals with $\mathrm{KOA}$ is described in clinical trials 
published in two systematic reviews. ${ }^{15} 16$ In general, both reviews found that dry cupping associated with drug therapy is superior to the exclusive use of the latter and improves the function of individuals with KOA. However, the studies included exhibit poor methodological quality, thereby compromising the use of this resource in clinical practice. The following biases were identified: lack of randomisation and rater and participant blinding; absence of a follow-up period; not comparing with a sham dry cupping group; not including functional tests to assess the physical performance of individuals with $\mathrm{KOA}$, as recommended for clinical trials with $\mathrm{KOA},{ }^{21}$ and no control over the use of analgesics during the interventions. ${ }^{15} 16$

Well-designed studies with high methodological quality are needed before any final conclusion can be drawn about the efficacy of dry cupping in individuals with KOA. Our study will be designed with high methodological quality, with all the study procedures clearly described: considerable sample size, adequate sample randomisation, blinding of participants, the assessor and researcher in charge of statistical analyses, 6 -week intervention and a 1-month follow-up to better assess therapy efficacy. The study involves four assessments, where pain will be evaluated both at rest and after functional tests. In addition, the use of any therapeutic resource during the interventions will be controlled and intention-to-treat analysis applied.

This study will follow the recommendations used in earlier research that applied sham dry cupping therapy. ${ }^{1933}$ In order to compare participant blinding, we will include people who have no prior experience with dry cupping, in addition to placing an opaque structure transversally on the subjects' thigh to prevent them from seeing the technique being applied. Moreover, participants will complete questionnaires and undergo the functional tests wearing pants, and with the knee region covered during all the assessments, so that the rater will not know to which group the subject was allocated. Application times of the different interventions will also be different, to avoid contact between participants of the different groups. At the end of the last intervention, subjects will be asked which treatment technique they believe they received (real or sham).

A limitation of our study is the unrestricted use of other therapeutic modalities by participants to reduce pain during the study period. In order to minimise this limitation, participants will be asked before each intervention whether they made use of any therapeutic modality for analgesia (medication or not) between interventions. This information will be recorded before each intervention and analysed at the end of the 12 protocol sessions as well as in the 1-month follow-up. The fact that the therapist who will deliver dry cupping or the sham intervention will not be blinded can also be considered a limitation of this study. Finally, participants will not present with radiographically confirmed KOA. They will, however, meet the ACR clinical criteria and have a minimum level of pain in order to ensure the possibility for improvement with the interventions.

There is no scientific evidence to support the use of dry cupping in clinical practice, or consensus regarding the application technique of this intervention in individuals with KOA. This is the first randomised blind placebo-controlled clinical trial that will serve as the basis for other studies, in order to provide a better scientific framework for the clinical use of dry cupping in individuals with KOA.

\section{Ethics and dissemination}

This protocol was approved by the UFRN/FACISA Research Ethics Committee (number: 3.737.688). There will be previous contact with participants on social media to inform them of the study objectives and resolution no. 466/2012 of the National Health Council of Brazil, which standardised regulatory guidelines for research involving human beings. The results will be submitted to specialised peer-reviewed journals and scientific meetings.

\section{Trial status}

Volunteers had yet to be recruited at the time of manuscript submission.

Correction notice This article has been corrected since it first published. The provenance and peer review statement has been included.

Contributors Study conception and design: NSP, GMB, HJAS, RSS, CAdAL, MCS. Data analysis planning: GMB, CAdAL, MCS. Drafting of the manuscript: NSP, GMB, HJAS, RSS, CAdAL, CGS, MCS. Critical revisions: all authors. Final approval of the article: all authors. Obtaining funding: MCS, responsibility for the integrity of the work as a whole.

Funding This study was financed in part by the Coordenação de Aperfeiçoamento de Pessoal de Nível Superior-Brasil (CAPES)_Finance Code 001. This study was performed with the authors' own resources and no private funding.

Competing interests None declared.

Patient and public involvement Patients and/or the public were involved in the design, or conduct, or reporting, or dissemination plans of this research. Refer to the Methods section for further details.

Patient consent for publication Obtained.

Provenance and peer review Not commissioned; externally peer reviewed.

Open access This is an open access article distributed in accordance with the Creative Commons Attribution Non Commercial (CC BY-NC 4.0) license, which permits others to distribute, remix, adapt, build upon this work non-commercially, and license their derivative works on different terms, provided the original work is properly cited, appropriate credit is given, any changes made indicated, and the use is non-commercial. See: http://creativecommons.org/licenses/by-nc/4.0/.

\section{ORCID iDs}

Nayara Silva Pontes http://orcid.org/0000-0002-4955-5563 Germanna Medeiros Barbosa http://orcid.org/0000-0002-1094-334X Hugo Jário Almeida Silva http://orcid.org/0000-0003-2185-4059 Rodrigo Scattone Silva http://orcid.org/0000-0002-7973-188X Clécio Gabriel Souza http://orcid.org/0000-0001-9005-7956

Caio Alano de Almeida Lins http://orcid.org/0000-0001-6424-3114

Marcelo Cardoso de Souza http://orcid.org/0000-0002-9268-8353

\section{REFERENCES}

1 Lamoth C, van Deudekom FJ, Van Campen J. Gait characteristics and their discriminative power in geriatric patients with and without cognitive impairment. J Neuroeng Rehabil 2017;14. 
2 Bennell KL, Dobson F, Hinman RS. Exercise in osteoarthritis: moving from prescription to adherence. Best Pract Res Clin Rheumatol 2014;28:93-117.

3 GBD 2016 DALYs and HALE Collaborators. Global, regional, and national disability-adjusted life-years (DALYs) for 333 diseases and injuries and healthy life expectancy (HALE) for 195 countries and territories, 1990-2016: a systematic analysis for the global burden of disease study 2016. Lancet 2017;390:1260-344.

4 Zhang W, Nuki G, Moskowitz RW, et al. OARSI recommendations for the management of hip and knee osteoarthritis: Part III: changes in evidence following systematic cumulative update of research published through January 2009. Osteoarthritis Cartilage 2010;18:476-99.

5 Miller GD, Jenks MZ, Vendela M, et al. Influence of weight loss, body composition, and lifestyle behaviors on plasma adipokines: a randomized weight loss trial in older men and women with symptomatic knee osteoarthritis. J Obes 2012;2012:1-14

6 Stevenson JD, Roach R. The benefits and barriers to physical activity and lifestyle interventions for osteoarthritis affecting the adult knee. $J$ Orthop Surg Res 2012;7:15.

7 Vincent HK, Heywood K, Connelly J, et al. Obesity and weight loss in the treatment and prevention of osteoarthritis. Pm R 2012;4:S59-67.

8 McAlindon TE, Bannuru RR, Sullivan MC, et al. OARSI guidelines for the non-surgical management of knee osteoarthritis. Osteoarthritis Cartilage 2014;22:363-88.

9 Fernandes L, Hagen KB, Bijlsma JWJ, et al. EULAR recommendations for the non-pharmacological core management of hip and knee osteoarthritis. Ann Rheum Dis 2013;72:1125-35.

10 Hochberg MC, Altman RD, April KT, et al. American College of rheumatology 2012 recommendations for the use of nonpharmacologic and pharmacologic therapies in osteoarthritis of the hand, hip, and knee. Arthritis Care Res 2012;64:465-74.

11 Jevsevar DS. Treatment of osteoarthritis of the knee: evidence-based guideline, 2nd edition. J Am Acad Orthop Surg 2013;21:571-6.

12 Al-Bedah A, Aboushanab TS, Alqaed M, et al. Classification of cupping therapy: a tool for modernization and standardization. JOCAMR 2016;1:1-10.

13 Rozenfeld E, Kalichman L. New is the well-forgotten old: the use of dry cupping in musculoskeletal medicine. J Bodyw Mov Ther 2016;20:173-8.

14 Markowski A, Sanford S, Pikowski J, et al. A pilot study analyzing the effects of Chinese cupping as an adjunct treatment for patients with subacute low back pain on relieving pain, improving range of motion, and improving function. J Altern Complement Med 2014;20:113-7.

15 Li J-Q, Guo W, Sun Z-G, et al. Cupping therapy for treating knee osteoarthritis: the evidence from systematic review and metaanalysis. Complement Ther Clin Pract 2017;28:152-60.

16 Wang Y-L, An C-M, Song S, et al. Cupping therapy for knee osteoarthritis: a synthesis of evidence. Complement Med Res 2018;25:249-55

17 Lauche $\mathrm{R}$, Cramer $\mathrm{H}$, Hohmann $\mathrm{C}$, et al. The effect of traditional cupping on pain and mechanical thresholds in patients with chronic nonspecific neck pain: a randomised controlled pilot study. Evid Based Complement Alternat Med 2012;2012:1-10.

18 Aboushanab TS, AlSanad S. Cupping therapy: an overview from a modern medicine perspective. J Acupunct Meridian Stud 2018;11:83-7.

19 Silva HJdeA, Saragiotto BT, Silva RS, et al. Dry cupping in the treatment of individuals with non-specific chronic low back pain: a protocol for a placebo-controlled, randomised, double-blind study. BMJ Open 2019:9:e032416.

20 Schulz KF, Altman DG, Moher D, et al. Consort 2010 statement: updated guidelines for reporting parallel group randomised trials. BMJ 2010;340:c332.

21 McAlindon TE, Driban JB, Henrotin Y, et al. OARSI clinical trials recommendations: design, conduct, and reporting of clinical trials for knee osteoarthritis. Osteoarthritis Cartilage 2015;23:747-60.

22 Zhang X, Tian R, Lam WC, et al. Standards for reporting interventions in clinical trials of cupping (STRICTOC): extending the CONSORT statement. Chin Med 2020;15:10.

23 Chan A, Tetzlaff JM, Altman DG. Declaración SPIRIT 2013: definición de los elementos estándares del protocolo de un ensayo clínico [SPIRIT 2013 Statement: defining standard protocol items for clinical trials]. Rev Panam Salud Publica 2015;38:506-14.

24 Hoffmann TC, Glasziou PP, Boutron I, et al. Better reporting of interventions: template for intervention description and replication (TIDieR) checklist and guide. BMJ 2014;348:91687.

25 Peat G, Thomas E, Duncan R, et al. Clinical classification criteria for knee osteoarthritis: performance in the general population and primary care. Ann Rheum Dis 2006;65:1363-7.
26 Altman R, Asch E, Bloch D, et al. Development of criteria for the classification and reporting of osteoarthritis: classification of osteoarthritis of the knee. Arthritis \& Rheumatism 1986;29:1039-49.

27 Hawker GA, Mian S, Kendzerska T, et al. Measures of adult pain: visual analog scale for pain (vas pain), numeric rating scale for pain (NRS pain), McGill pain questionnaire (MPQ), short-form McGill pain questionnaire (SF-MPQ), chronic pain grade scale (CpGs), short Form-36 bodily pain scale (SF. Arthritis Care Res 2011;63:S240-52.

28 Dantas LO, Breda CC, da Silva Serrao PRM, et al. Short-Term cryotherapy did not substantially reduce pain and had unclear effects on physical function and quality of life in people with knee osteoarthritis: a randomised trial. J Physiother 2019;65:215-21.

29 Wolfe F, Clauw DJ, Fitzcharles M-A, et al. The American College of rheumatology preliminary diagnostic criteria for fibromyalgia and measurement of symptom severity. Arthritis Care Res 2010;62:600-10.

30 Vassão PG, Silva BA, de Souza MC, et al. Level of pain, muscle strength and posture: effects of PBM on an exercise program in women with knee osteoarthritis - a randomized controlled trial. Lasers Med Sci 2020;35:1967-74.

31 Uysal A, Yildizgoren MT, Guler H, et al. Effects of radial extracorporeal shock wave therapy on clinical variables and isokinetic performance in patients with knee osteoarthritis: a prospective, randomized, single-blind and controlled trial. Int Orthop 2020;44:1311-9.

32 Chen H, Wang Y, Liu C, et al. Benefits of a transtheoretical modelbased program on exercise adherence in older adults with knee osteoarthritis: a cluster randomized controlled trial. J Adv Nurs 2020;76:1765-79.

33 Lee MS, Kim J-I, Kong JC, et al. Developing and validating a sham cupping device. Acupunct Med 2010;28:200-4.

34 Kamper S. Global rating of change scales. Aust J Physiother 2009;55:289.

35 Tham LM, Lee HP, Lu C. Cupping: from a biomechanical perspective. J Biomech 2006;39:2183-93.

36 Foster NE, Thomas E, Barlas P, et al. Acupuncture as an adjunct to exercise based physiotherapy for osteoarthritis of the knee: randomised controlled trial. BMJ 2007;335:436-40.

37 Witt C, Brinkhaus B, Jena S, et al. Acupuncture in patients with osteoarthritis of the knee: a randomised trial. Lancet 2005;366:136-43.

38 Teut M, Kaiser S, Ortiz M, et al. Pulsatile dry cupping in patients with osteoarthritis of the knee - a randomized controlled exploratory trial. BMC Complement Altern Med 2012;12:184.

39 Trial ATR, Scharf H, Mansmann U. Annals of internal medicine article acupuncture and knee osteoarthritis. Ann Intern Med 2006;145:1-16.

40 Stokes V. A manual of acupuncture. Physiotherapy 1998;84:458.

41 Sun N, Shi G-X, Tu J-F, et al. Traditional Chinese acupuncture versus minimal acupuncture for mild-to-moderate knee osteoarthritis: a protocol for a randomised, controlled pilot trial. BMJ Open 2016;6:e013830.

42 Fernandes MI. Translation and validation of the specific quality of life questionnaire for osteoarthritis WOMAC (Western Ontario and McMaster universities) for Portuguese language. Osteoarthritis and Cartilage 2002;12:101-68.

43 Kirkwood RN, Resende RA, Magalhães CMB, et al. Application of principal component analysis on gait kinematics in elderly women with knee osteoarthritis. Rev Bras Fisioter 2011;15:52-8.

44 Wright AA, Cook CE, Baxter GD, et al. A comparison of 3 methodological approaches to defining major clinically important improvement of 4 performance measures in patients with hip osteoarthritis. J Orthop Sports Phys Ther 2011;41:319-27.

45 Ciconelli RM, Ferraz MB, Santos W. Brazilian-Portuguese version of the SF-36. A reliable and valid quality of life outcome measure. Rev Bras Reumatol 1997;39:143-50.

46 Beasley MJ, Ferguson-Jones EA, Macfarlane GJ. Treatment expectations but not preference affect outcome in a trial of CBT and exercise for pain. Can J Pain 2017;1:161-70.

47 Freitas P, Pires D, Nunes C. Cross-Cultural adaptation and psychometric properties of the European Portuguese version of the global perceived effect scale in patients with chronic low back pain cross-cultural adaptation and psychometric properties of the European low back pain. Disabil Rehabil 2019;0:1-7.

48 Costa LOP, Maher CG, Latimer J, et al. Clinimetric testing of three self-report outcome measures for low back pain patients in Brazil: which one is the best? Spine 2008;33:2459-63.

49 Draper DO, Klyve D, Ortiz R, et al. Effect of low-intensity longduration ultrasound on the symptomatic relief of knee osteoarthritis: a randomized, placebo-controlled double-blind study. J Orthop Surg Res 2018:13:257. 